\title{
Effect of Basic Drive Train Cycle Length on Induction of Ventricular Tachycardia by a Single Extrastimulus
}

\author{
FRED MORADY, M.D., WILLIAM H. KOU, M.D., ALAN H. KADISH, M.D., \\ STEPHEN SCHMALTZ, M.P.H., JONI SUMMITT, D.O., and \\ SHIMON ROSENHECK, M.D.
}

From the Division of Cardiology and the Clinical Research Center, Department of Internal Medicine, University of Michigan Medical Center, Ann Arbor, Michigan

MORADY F, ET AL: Effect of Basic Drive Train Cycle Length on Induction of Ventricular Tachycardia by a Single Extrastimulus. This study determined the effects of a wide range of basic drive cycle lengths on the induction of ventricular tachycardia (VT) by a single extrastimulus $\left(S_{2}\right)$. Seventy-one patients with coronary artery disease and inducible sustained monomorphic VT underwent 121 electrophysiology tests either in the control state or during treatment with an antiarrhythmic drug. Ventricular basic drive trains were eight beats in duration and the intertrain interval was three seconds. Programmed ventricular stimulation was performed with $S_{2}$ using the longest possible basic drive cycle length rounded off to the nearest multiple of $100 \mathrm{msec}$, then using basic drive train cycle lengths that decreased in $100 \mathrm{msec}$ steps to $400 \mathrm{msec}$, and finally using a basic drive cycle length of $350 \mathrm{msec}$. At each drive cycle length, an interval of $>50$ msec beyond the effective refractory period (ERP) was scanned with $S_{2}$. Monomorphic VT was induced by $\mathrm{S}_{2}$ in 52/121 studies (43\%). The drive cycle length had a significant linear effect on the log odds of inducing VT $(P<0.0001)$. The highest yield of VT occurred with a drive cycle length of $350 \mathrm{msec}(42 / 121,34 \%)$, and with each increment in drive cycle length, the expected odds of inducing $V T$ decreased by a factor of 1.7. In $88 \%$ of cases in which VT was induced at a particular drive cycle length but not at longer drive cycle lengths, the coupling intervals that induced VT exceeded the ERP measured at one or more of the longer basic drive cycle lengths. In conclusion, there is an inverse relationship between the basic drive cycle length and the yield of monomorphic VT induced by $S_{2}$. The use of shorter basic drive cycle lengths often facilitates the induction of VT by some effect other than critical shortening of the $S_{2}$ coupling interval. (J Electrophysiol 3:111-116, 1989)

programmed ventricular stimulation, ventricular tachycardia

Programmed ventricular stimulation typically is performed using basic drive trains that vary in cycle length between 400 and $600 \mathrm{msec}^{1-9}$ The cumulative yield of ventricular tachycardia (VT) induced by single or double ventricular extrastimuli has been demonstrated to increase

Supported in part by Grant 5M01-RR00042-25 from the National Institutes of Health, Bathesda, Maryland.

Address for correspondence: Fred Morady, M.D., The University of Michigan Hospital, 1500 East Medical Center Drive, Cardiology B1F245-0022, Ann Arbor, MI 48109. with the use of multiple basic drive cycle lengths spanning a range of at least 200 msec. ${ }^{10}$ However, no prior studies have compared the inducibility of VT at various basic drive cycle lengths. The purpose of this prospective study was to determine the effects of a wide range of basic drive cycle lengths on the induction of VT by a single extrastimulus. The study protocol was limited to the use of a single extrastimulus in order to allow a direct comparison of the coupling intervals required to induce VT at different basic drive cycle lengths. 


\section{Methods}

\section{Selection of Subjects}

The following criteria were used to select the subjects for this study: (1) presence of coronary artery disease; (2) sinus rhythm; (3) a documented history of sustained, monomorphic VT; (4) sustained monomorphic VT inducible by programmed ventricular stimulation with 1, 2, or 3 extrastimuli; (5) hemodynamic stability during induced VT; and (6) ability to terminate induced VT by pacing. Because analysis of the effects of the basic drive cycle length on the induction of VT would be confounded by the induction of various types of VT, patients in whom more than one configuration of VT was induced by a single extrastimulus were excluded from this study. Also excluded were patients in whom induced VT was preceded by a bundle branch reentry beat, since the branch reentry beats may have served as a second extrastimulus.

\section{Characteristics of Patients}

Seventy-one patients with coronary artery disease met the inclusion criteria for this study. There were 67 men and 4 women, and their mean age was $64 \pm 10$ years ( \pm standard deviation). The mean left ventricular ejection fraction, determined in 45 patients, was $31 \pm 9$. In 10 patients, the study protocol was performed only in the absence of antiarrhythmic drug therapy, in 17 patients the protocol was performed in both the control state and during antiarrhythmic drug therapy, and in 44 patients the study protocol was performed only during antiarrhythmic drug therapy because the induced VT was not hemodynamically stable in the control state. The antiarrhythmic drugs that were used included quinidine in 19 studies, mexiletine in 11 studies, quinidine plus mexiletine in 3 studies, encainide in 14 studies, amiodarone in 31 studies, and a combination of amiodarone plus one of the other antiarrhythmic drugs in 16 studies. Data from a total of 121 electrophysiology tests are included in this study.

\section{Study Protocol}

Electrophysiology studies were performed in the fasting, nonsedated state after informed consent was obtained. Three $6 \mathrm{~F}$ quadripolar electrode catheters were inserted into a femoral vein and positioned in the right atrium, His bundle position, and right ventricular apex. A short $5 \mathrm{~F}$ cannula inserted into a femoral artery was used to monitor the arterial pressure. Leads $\mathrm{V}_{1}$, I and III, and the intracardiac electrograms were displayed in an oscilloscope and recorded with a Siemens-Elema Mingograf-7 recorder (Siemens-Elema, Solna, Sweden). Pacing was performed using a programmable stimulator (Bloom Associates, Ltd., Narbeth, PA, USA). The pacing stimuli were twice the diastolic threshold and $2 \mathrm{msec}$ in duration.

Basic drive trains were eight beats in duration and separated by pauses of three seconds. Extrastimuli were scanned in steps of $10 \mathrm{msec}$. If there was atrioventricular dissociation during ventricular pacing, the atrium was paced synchronously with the ventricle in order to prevent interruption of the basic drive train by sinus capture beats.

The study protocol was performed upon completion of the standard, clinically used stimulation protocol, that consisted of programmed stimulation with 1,2 , and 3 ventricular extrastimuli using basic drive cycle lengths of 600 and $400 \mathrm{msec}$, first at the right ventricular apex, then if necessary, at the right ventricular outflow tract or septum. Sustained VT was defined as VT lasting $>30$ seconds or requiring countershock or pacing to terminate; nonsustained VT was defined as VT 6 beats to 30 seconds in duration. In accord with the selection criteria for subjects, sustained monomorphic VT was inducible by 1,2 , or 3 extrastimuli in every patient in this study.

Because of time constraints, the study protocol was performed only at the right ventricular apex. Diastole was scanned with an extrastimulus during ventricular pacing, using the longest possible basic drive cycle length rounded off to the nearest multiple of $100 \mathrm{msec}$. Programmed stimulation with the single extrastimulus then was repeated using basic drive 
train cycle lengths that decreased in $100 \mathrm{msec}$ steps to $400 \mathrm{msec}$, and finally using a basic drive cycle length of $350 \mathrm{msec}$. At each basic drive cycle length, an interval of at least $50 \mathrm{msec}$ beyond the effective refractory period (ERP) was scanned with the extrastimulus. Whenever VT was induced, programmed stimulation was continued at the same basic drive cycle length until all of the coupling intervals that resulted in the induction of VT were identified. The endpoint of the study protocol was completion of programmed stimulation using all of the basic drive train cycle lengths. If at any point in the protocol a hemodynamically unstable VT requiring countershock was induced, the study protocol was stopped and the patient was excluded from the study.

\section{Data Analysis}

To study the relationship between ventricular ERP and the basic drive cycle length, a random coefficient growth curve model was fit to the data. ${ }^{11}$ For each of the 121 cases in the study, a regression of ERP on basic drive cycle length was fit. The 121 slopes and intercepts obtained from these regression lines were then analyzed. The effect of the basic drive cycle length on the induction of VT by a single extrastimulus was analyzed using logistic regression. ${ }^{12}$ For this analysis, the log odds of inducing VT was modeled as a linear function of the basic drive cycle length. The effect of the basic drive cycle length on the range of coupling intervals that induced VT was analyzed using an analysis of variance. A $P$ value $<0.05$ was considered statistically significant.

\section{Results}

\section{Ventricular Effective Refractory Periods}

The mean ventricular ERPs are listed in Table 1. The basic drive cycle length had a significant linear effect on ERP $(P<0.0001)$, with each decrement in drive cycle length decreasing the expected value of the ERP by $11 \mathrm{msec}$.

\section{Induction of VT by One Extrastimulus}

Monomorphic VT was induced by a single extrastimulus in a total of 52/121 electrophysiology studies (43\%). The VT was sustained in 48 of the studies and nonsustained in four. Polymorphic VT was never induced. Table 1 displays the incidence of monomorphic VT induced by one extrastimulus at each basic drive cycle length. The incidence of induced VT was highest when the basic drive cycle length was $350 \mathrm{msec}$ (41/121, 34\%). Among 52 studies in

TABLE 1

Effect of Basic Drive Cycle Length on Ventricular Refractoriness and Induction of Ventricular Tachycardia by a Single Extrastimulus

\begin{tabular}{|c|c|c|c|c|c|}
\hline \multirow[t]{2}{*}{$\begin{array}{l}\text { Basic } \\
\text { Drive Cycle } \\
\text { Length (ms) }\end{array}$} & $\begin{array}{l}\text { No. of } \\
\text { Studies }\end{array}$ & ERP (ms) & $\begin{array}{c}\text { No. With } \\
\text { Inducible VT }\end{array}$ & $\begin{array}{c}\text { Range of Cl's } \\
\text { That Induced VT: }\end{array}$ & \\
\hline & & & & Range (ms) & Sample Size \\
\hline 350 & 121 & $244 \pm 23+$ & $41(34 \%)$ & $43 \pm 24$ & 25 \\
\hline 400 & 121 & $253 \pm 23$ & $34(28 \%)$ & $41 \pm 24$ & 25 \\
\hline 500 & 121 & $263 \pm 22$ & $19(16 \%)$ & $40 \pm 19$ & 17 \\
\hline 600 & 121 & $274 \pm 24$ & $10(8 \%)$ & $38 \pm 35$ & 8 \\
\hline 700 & 107 & $286 \pm 25$ & $6(6 \%)$ & $45 \pm 34$ & 6 \\
\hline 800 & 83 & $300 \pm 24$ & $5(6 \%)$ & $27 \pm 15$ & 3 \\
\hline 900 & 48 & $314 \pm 29$ & $2(4 \%)$ & - & 0 \\
\hline 1000 & 24 & $318 \pm 32$ & $1(4 \%)$ & - & 0 \\
\hline 1100 & 15 & $323 \pm 25$ & 0 & - & 0 \\
\hline
\end{tabular}

+ Mean \pm standard deviation

"There were no significant differences between the various basic drive cycle lengths.

$\mathrm{Cl}=$ coupling interval, ERP = effective refractory period, No. = number, $\mathrm{VT}=$ ventricular tacyhcardia. 
which VT was induced by one extrastimulus, $350 \mathrm{msec}$ was the only basic drive cycle length at which VT was induced in 15 (29\%). The drive cycle length had a significant linear effect on the log odds of inducing VT $(\mathrm{P}<0.0001)$, with each increment in drive cycle length decreasing the expected odds of inducing VT by a factor of 1.7.

The mean cycle length of VT induced by one extrastimulus, $427 \pm 64 \mathrm{msec}$, was significantly longer than the mean cycle length of VT inducible only by 2 or 3 extrastimuli, $352 \pm 80 \mathrm{msec}$ $(P<0.001)$. Among the 69 electrophysiology studies in which VT was not inducible with a single extrastimulus, monomorphic VT was induced by two or three extrastimuli in 44 and 25 studies, respectively. A similar proportion of VTs induced by one versus two or three extrastimuli had a right bundle branch block configuration ( $60 \%$ vs $58 \%$, respectively).

\section{Coupling Intervals at Which VT Was Induced}

Whenever $\geq 2$ coupling intervals induced VT at a particular basic drive cycle length, the range of coupling intervals that induced VT was determined; the mean range of extrastimulus coupling intervals that induced VT at each basic drive cycle length are listed in Table 1 . The range of coupling intervals that induced VT did not differ significantly among the various drive cycle lengths. When VT was inducible at a particular drive cycle length, the shortest coupling interval that induced VT was within $10 \mathrm{msec}$ of the ventricular ERP in $52 \%$ of cases, and > $20 \mathrm{msec}$ beyond the ventricular ERP in the remaining cases.

In $88 \%$ of cases, when VT was induced at a particular drive cycle length but not at longer drive cycle lengths, the coupling intervals that induced VT exceeded the ERP measured at 1 or more of the longer basic drive cycle lengths.

\section{Effect of Antiarrhythmic Drug Therapy}

A comparison of electrophysiology tests performed in the control state and those performed in the presence of antiarrhythmic drug therapy demonstrated no significant difference in the relative yield of VT at the various basic drive cycle lengths.

\section{Discussion}

\section{Yield of Ventricular Tachycardia with a Single Extrastimulus}

Among 121 cases of sustained monomorphic VT inducible by programmed stimulation with 1-3 extrastimuli, the use of multiple basic drive cycle lengths resulted in a $43 \%$ yield of inducible VT when a single extrastimulus was used. In contrast, in prior clinical studies that have used only two basic drive cycle lengths between 600 and $400 \mathrm{msec}$, the percentage of monomorphic VTs induced by a single extrastimulus has been $16 \%-28 \% .^{2,4,5,7,9}$ In the present study, had the basic drive cycle lengths been limited to 400 and $600 \mathrm{msec}$, as is typical in clinical stimulation protocols, the yield of VT with a single extrastimulus would have been $28 \%$. Therefore, the use of multiple basic drive cycle lengths increases the yield of monomorphic VT during programmed stimulation with one extrastimulus.

\section{Effect of Basic Drive Cycle Length}

The results of this study demonstrate an inverse relationship between the basic drive cycle length and the yield of monomorphic VT induced by a single extrastimulus. The shortest basic drive cycle length used in this study ( 350 $\mathrm{msec}$ ) resulted in a $34 \%$ yield of VT during programmed stimulation with one extrastimulus, and there was a progressive fall in the yield of VT as the basic drive cycle length increased.

\section{Coupling Intervals Associated with VT Induction}

As expected, there was a progressive shortening of the ventricular ERP as the basic drive cycle length decreased. Therefore, the shorter basic drive cycle lengths allowed the use of extrastimulus coupling intervals that would not have resulted in ventricular capture at longer 
basic drive cycle lengths, and this may have been a factor accounting for the higher yield of VT at the shorter basic drive cycle lengths. However, in more than $80 \%$ of cases, VT was induced by coupling intervals that exceeded the ERP at longer basic drive cycle lengths and that nevertheless had not induced VT at the longer drive cycle lengths. Therefore, the use of shorter basic drive cycle lengths often facilitates the induction of VT by some effect independent of the extrastimulus coupling interval. As has been suggested previously, ${ }^{13}$ shortening the basic drive cycle length may facilitate the induction of VT by resulting in more slowing of conduction and/or greater heterogeneity in refractory periods within the reentrant circuit.

\section{Comparison with Prior Studies}

In this study, the basic drive cycle length did not have an effect on the range of coupling intervals that induced VT. In contrast, Breithardt et al. $^{13}$ reported that a decrease in the basic drive cycle length resulted in a widening in the range of coupling intervals capable of inducing VT. These disparate results may be attributable to differences in study design. Breithardt et al. ${ }^{13}$ compared the sum of coupling intervals that induced VT with one or two extrastimuli at basic drive cycle lengths of 500 or $430 \mathrm{msec}$ versus 370 or $330 \mathrm{msec}$. However, in the present study only one extrastimulus was used and the extrastimulus coupling intervals that induced VT were compared at individual drive cycle lengths ranging between 350 to $1100 \mathrm{msec}$.

\section{Limitations}

The subjects in this study were limited to patients who had hemodynamically-tolerated VT, and therefore the conclusions of the study may not apply in patients with rapid and hemodynamically unstable VT.

Many of the subjects in this study were being treated with antiarrhythmic drugs at the time of electrophysiological testing, and these drugs may have influenced the results. For example, it is possible that programmed stimulation with one extrastimulus is more likely to induce VT in the presence of antiarrhythmic drugs than in the control state. However, no difference in the relative yield of VT among the various drive cycle lengths was found when comparing control studies with studies performed during therapy with antiarrhythmic drugs.

The results of this study suggest that clinical programmed stimulation protocols should incorporate the use of a basic drive cycle length of $350 \mathrm{msec}$, because this will significantly increase the probability of inducing VT with one extrastimulus. The induction of monomorphic VT by one extrastimulus not only shortens the duration of electrophysiological testing but also may improve specificity by obviating the need for programmed stimulation with multiple extrastimuli, which are more likely to induce nonclinical forms of VT. However, the relative sensitivity and specificity of stimulation with one extrastimulus at a basic drive cycle length of $350 \mathrm{msec}$ as compared to two and three extrastimuli at conventional drive cycle length of 400 and $600 \mathrm{msec}$ remains to be determined.

Acknowledgment: The authors are grateful to Judy Hanson for her excellent secretarial assistance.

\section{References}

1. Doherty JU, Kienzle MG, Waxman HL, et al: Relation of mode of induction and cycle length of ventricular tachycardia: analysis of 104 patients. Am J Cardiol, 52:60, 1983.

2. Mann DE, Luck JE, Griffin JC, et al: Induction of clinical ventricular tachycardia using programmed stimulation: value of third and fourth extrastimuli. Am J Cardiol, 52:501, 1983.

3. Doherty DU, Kienzle MG, Waxman HL, et al : Programmed ventricular stimulation at a second right ventricular site: an analysis of 100 patients, with special reference to sensitivity, specificity, and characteristics of patients with induced ventricular tachycardia. Am J Cardiol, 52:1184, 1983.

4. Buxton AE, Waxman HL, Marchlinski FE: Role of triple extrastimuli during electrophysiological study of patients with documented sustained ventricular tachyarrhythmias. Circulation 69(3):532, 1984.

5. Morady F, DiCarlo L, Winston S, et al: A prospective comparison of triple extrastimuli and left ventricular stimulation in studies of ventricular 
tachycardia induction. Circulation 70(1):52, 1984.

6. Brugada $\mathrm{P}$, Wellens HJJ: Comparsion in the same patient of two programmed ventricular stimulation protocols to induce ventricular tachycardia. Am J Cardiol 55:380, 1985.

7. Herre JM, Mann DE, Luck JC, et al : Effect of increased current, multiple pacing sites and number of extrastimuli on induction of ventricular tachycardia. Am J Cardiol 57:102, 1986.

8. Morady F, DiCarlo LA, Baerman JM, et al: Comparison of coupling intervals that induce clinical and nonclinical forms of ventricular tachycardia during programmed stimulation. Am J Cardiol 57:1269, 1986.

9. Lin HT, Mann DE, Luctk JC, et al: Prospective comparison of right and left ventricular stimula- tion for induction of sustained ventricular tachycardia. Am J Cardiol 59:559, 1987.

10. Estes $M$, Garan $H, M c$ Govern B, et al: Influence of drive cycle length during programmed stimulation on induction of ventricular arrhythmias: analysis of 403 patients. Am J Cardiol 57:108, 1986.

11. Schluchter MD: BMDP 5V-unbalanced repeated measures models with structured covariance matrices. 86:29, 1988.

12. Freeman DH. Applied Categorical Data Analysis. New York: Marcel Dekker, Inc, pp 237-274, 1987.

13. Breithardt G, Borggrefe M, Podczeck A, et al: Influence of the cycle length of basic drive on induction of sustained ventricular tachycardia associated with coronary artery disease. Am J Cardiol 60:1306, 1987. 
This document is a scanned copy of a printed document. No warranty is given about the accuracy of the copy. Users should refer to the original published version of the material. 